Research Article

\title{
Neck Flexion Angle Estimation during Walking
}

\author{
Duc Cong Dang, ${ }^{1}$ Quoc Khanh Dang, ${ }^{2}$ Young Joon Chee, ${ }^{3}$ and Young Soo Suh ${ }^{1}$ \\ ${ }^{1}$ Electrical Engineering Department, University of Ulsan, Ulsan, Republic of Korea \\ ${ }^{2}$ NAMU Inc., Ulsan, Republic of Korea \\ ${ }^{3}$ Biomedical Engineering Department, University of Ulsan, Ulsan, Republic of Korea \\ Correspondence should be addressed to Young Soo Suh; yssuh@ulsan.ac.kr
}

Received 8 June 2017; Accepted 19 October 2017; Published 28 December 2017

Academic Editor: Yeesock Kim

Copyright (C) 2017 Duc Cong Dang et al. This is an open access article distributed under the Creative Commons Attribution License, which permits unrestricted use, distribution, and reproduction in any medium, provided the original work is properly cited.

\begin{abstract}
Neck pain is recently known as the fourth leading cause of disability and the number of patients is apparently increasing. By analyzing the effect of gravitational force on inertial sensor attached to the neck, this study aims to investigate the head flexion posture during walking. The estimated angle is compared with the craniovertebral angle which is measured with an optical tracker. A total of twenty subjects with no history of neck pain or discomfort were examined by walking on the treadmill inside the working range of an optical tracker. In our laboratory settings, the neck flexion angle (NFA) may have a linear relationship with the craniovertebral angle (CVA) in both static case and constant speed walking case. Therefore, inertial sensor, which is lightweight, low cost, and especially free in movement, can be used instead of a camera system. Our proposed estimation method shows its flexibility and gives a result with the mean of absolute error of estimated neck angle varying from 0.48 to 0.58 degrees, which is small enough to use in applications.
\end{abstract}

\section{Introduction}

Neck pain is one of the most common musculoskeletal disorders (MSDs) among both the general and working population, with an annual prevalence rate exceeding $30 \%$, and the literature is mixed as to whether it peaks or plateaus in middle age [1]. According to [2], the head in normal posture weights about 10 to $12 \mathrm{lbs}$., but when the head flexed forward, the force seen by the neck increases to $27 \mathrm{lbs}$. at $15^{\circ}$, $40 \mathrm{lbs}$. at $30^{\circ}, 49 \mathrm{lbs}$. at $45^{\circ}$, and $60 \mathrm{lbs}$. at $60^{\circ}$. That huge amount of force increases loading in the joints and muscles of the cervical spine and can be a major contribution factor to neck and upper limb disorders after a prolonged period of time [3].

Recent studies show greater interest in the relationship between neck pain and digital devices, using situations such as computers at the workplace $[3,4]$ or handheld devices [5-7]. The widespread use of digital technology, including computers and mobile devices, is related to the prevalence of neck pain due to the head forward flexion posture. Users tend to maintain their poor head posture even with the existence of pain symptoms or neck discomfort [5].

Besides neck pain, it is said that pedestrians may face some risk while walking or crossing road since they tend to miss visual targets or sound indications when looking down the smartphone $[8,9]$. Therefore, it is important to investigate the head flexion posture during user walking.

The neck flexion angle (NFA) can be estimated by using force sensors placed on the front, left, and back side of the neck [10] or camera systems to observe the craniovertebral angle (CVA) using infrared markers attached on the neck $[11,12]$. Another effective way to measure the neck flexion angle was proposed by using motion sensors to analyze the gravitational force placed on the neck $[13,14]$. In previous studies, the neck flexion angle is estimated for static pose such as standing or sitting down. In this study, we used an inertial sensor to estimate the neck flexion angle (NFA) during walking. OptiTrack camera system is used to obtain the craniovertebral angle (CVA), which is used as ground truth value for the analysis. A preliminary result 


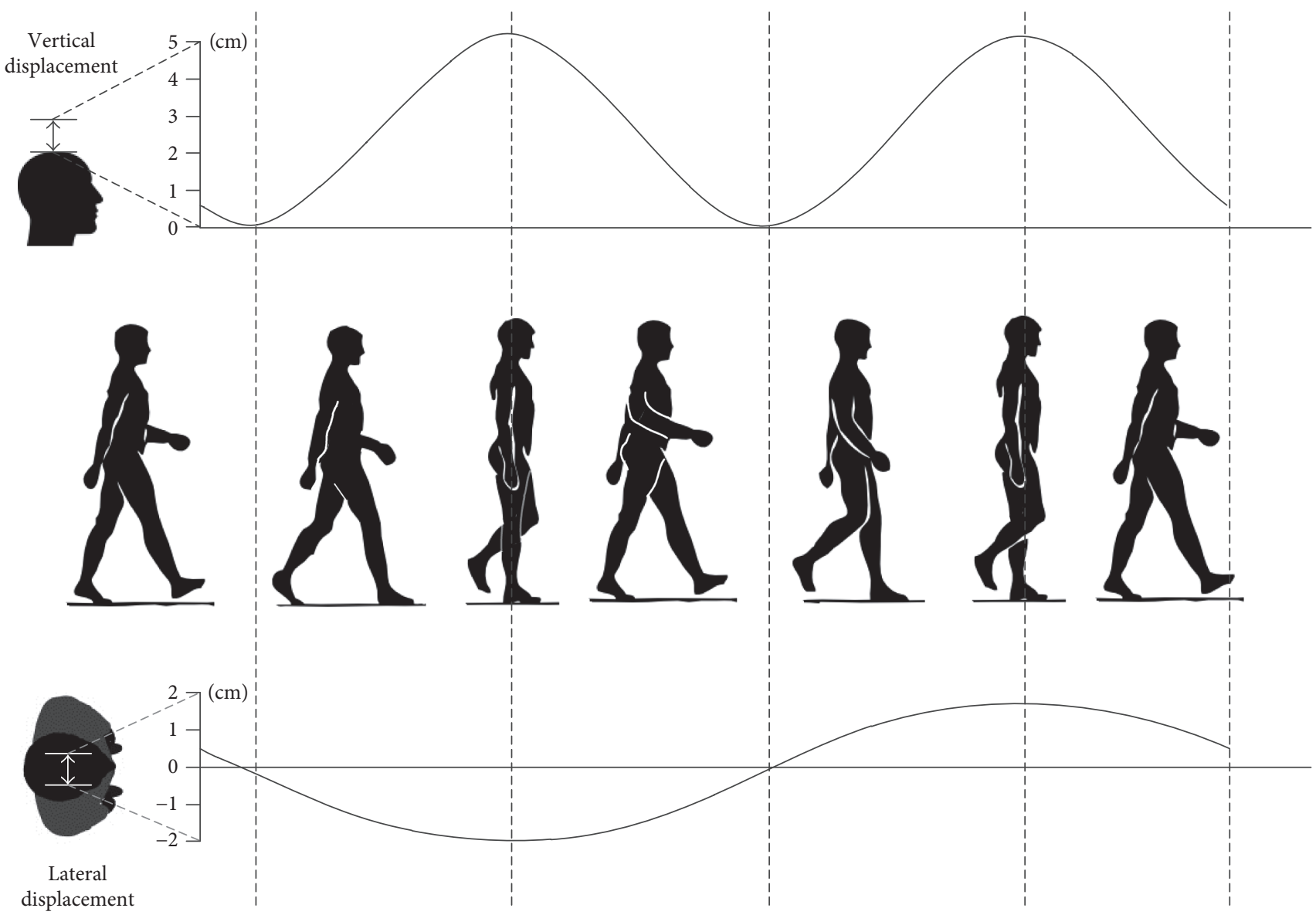

FIgURE 1: Vertical and lateral displacements of human gait.

was presented in the conference paper [15], where a basic algorithm is given without experimental verification.

\section{Methods}

The neck posture in this paper is estimated by analyzing the gravitational force placed on the neck using an accelerometer. During walking, the estimation is affected heavily by the walking movement since the accelerometer observes not only the gravitational force but also the external acceleration. The walking movement will be analyzed in this section together with the step detecting algorithm to propose a novel method for detecting neck angle during walking.

2.1. Human Gait Analysis. Human gait refers to locomotion achieved through the movement of human limb. During walking, the center of mass moves along the travelling direction but also has sinusoidal movements in the lateral and vertical direction [16]. The result in [16] also showed that the lateral and vertical displacements depend on the walking speed. When the nominal speed increases, the lateral displacement decreases while the vertical displacement tends to increase. However, according to a recorded data using VICON camera system from Carnegie Mellon University [17], the vertical and lateral translations are still in sinusoidal forms where the vertical movement frequency is double the lateral movement frequency.

The human gait analysis can be seen from Figure 1. As the lower limbs act like inverted pendulums while walking, the center of mass of a pedestrian moves in sinusoidal wave form with respect to the sagittal plane. The maximum vertical displacement is around $30 \%$ and $80 \%$ of gait cycle when one leg is at mid-stance and mid-swing positions, respectively. In the meantime, the sinusoidal form of the lateral displacement is caused by the changing of center of mass onto each foot while walking. The minimum of the lateral displacement is at $30 \%$ of the gait cycle while its maximum is at $80 \%$ of the gait cycle.

Based on this fact, a simulated movement can be generated as in Figure 2 where the forward movement is assumed to be linear. To verify the theory, a real data of a walking person was recorded. In this record, an accelerometer was attached on a volunteer's neck so that the $x$-axis of the accelerometer points upward while the $z$-axis of the accelerometer coincides with the walking direction. Since we are only interested in the form of the signal, the accelerometer outputs were filtered as can be seen from Figure 2(b). The result showed that while walking, the acceleration of the pedestrian's neck movement has both vertical and lateral accelerations in sinusoidal forms, in which the frequency of the later acceleration is twice that of the vertical acceleration. 

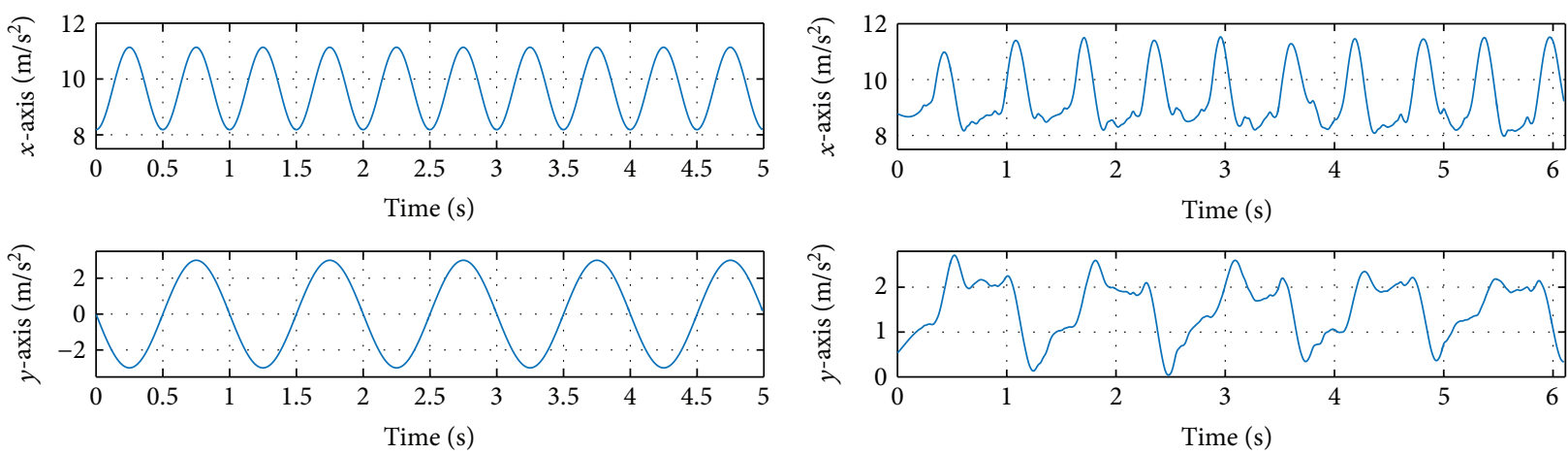

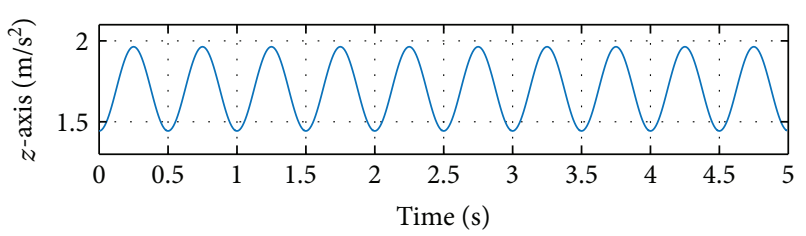

(a)

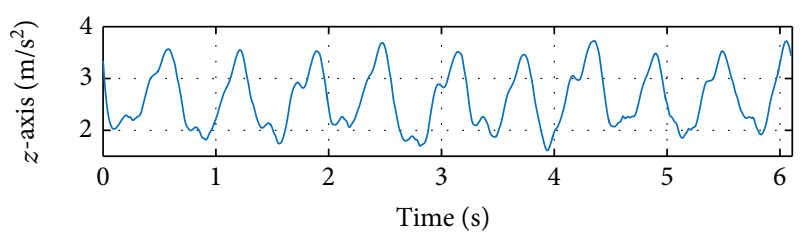

(b)

FIGURE 2: Simulated and real filtered accelerations of walking data: (a) simulated data; (b) real filtered data.

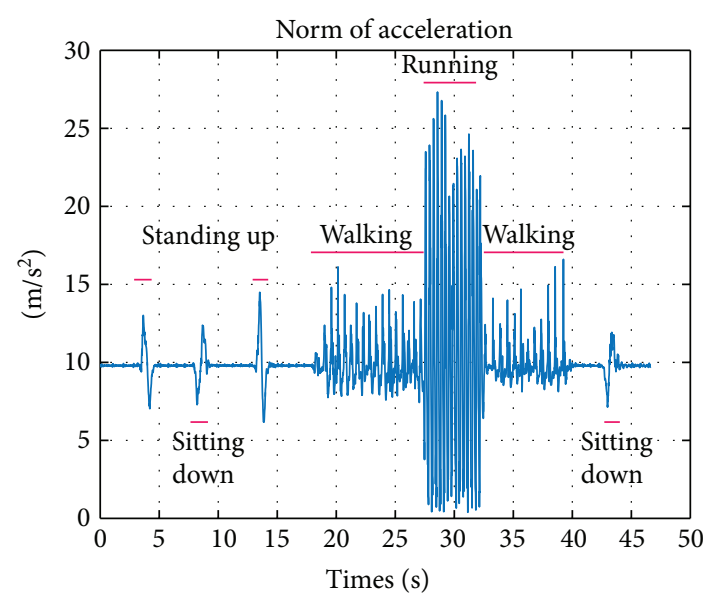

- Norm of acceleration

$\rightarrow$ Standard deviation of acceleration

(a)

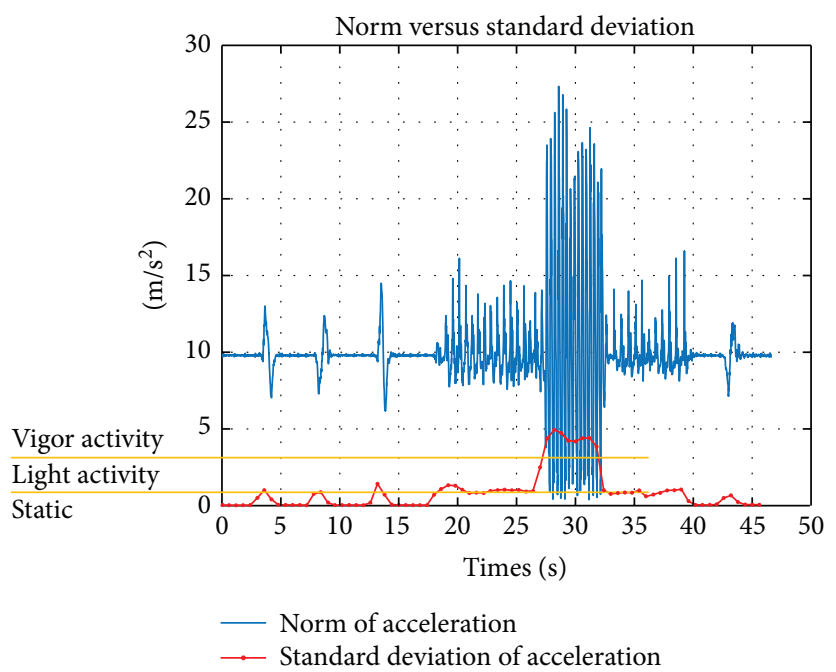

(b)

Figure 3: Activity classification using an accelerometer: (a) norm of acceleration; (b) norm and standard deviation of acceleration.

As can be seen from Figure 2, the peaks of the vertical acceleration were produced by the shocks yielded at the heel strike periods of the gait cycle. Based on these peaks, the steps can be detected. Since in each step the accelerations in vertical and lateral directions are close to sinusoidal forms and the forward acceleration is approximated to zero (linear forward walking), the external accelerations will be eliminated by summing the acceleration values in each axis. Or in other words, the sum of the acceleration in the $x$ - and $z$-axes in each step represents the gravitational force acting upon the pedestrian's neck. By only measuring the gravitational force, the accelerometer estimates the inclination angle of the accelerometer with respect to the ground using a triad algorithm given in [18]. This inclination is also the neck angle. Therefore, to cancel out the external acceleration, a step detection algorithm is needed.
2.2. Step Detection Algorithm. As mentioned above, the estimation of the neck angle is heavily affected by the external acceleration. In the first step, we aim to classify the human activities into dynamic and static cases using standard deviation of the acceleration as the main classification criteria. The standard deviation $\sigma_{a}$ of norm acceleration data set $a_{i}(1 \leq$ $i \leq N, N$ is the number of samples) is calculated as follows:

$$
\sigma_{a}=\sqrt{\frac{1}{N} \sum_{i=1}^{N}\left(a_{i}-\mu\right)^{2}},
$$

where $\mu=(1 / N) \sum_{i=1}^{N} a_{i}$ is the mean value of data set $a_{i}$.

By applying the standard deviation parameter to acceleration norm data, some human activities can be easily detected as an example in Figure 3, where a sequence 


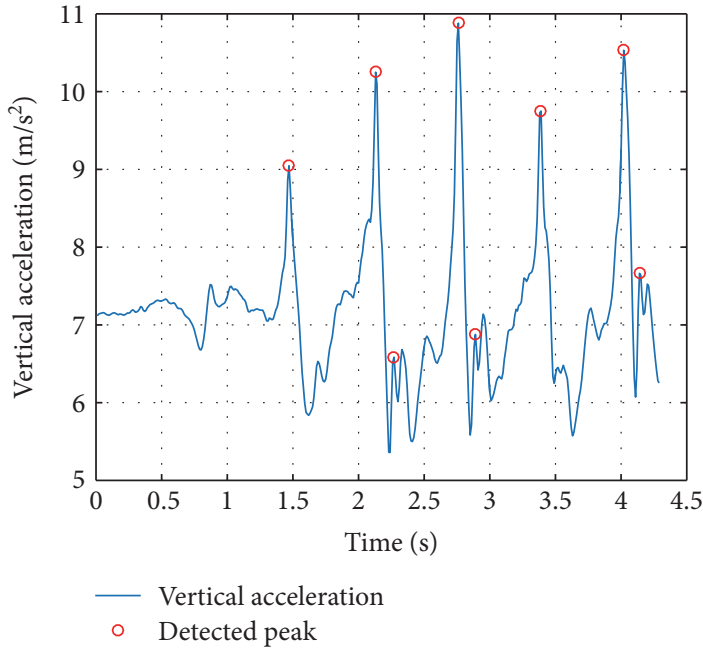

(a)

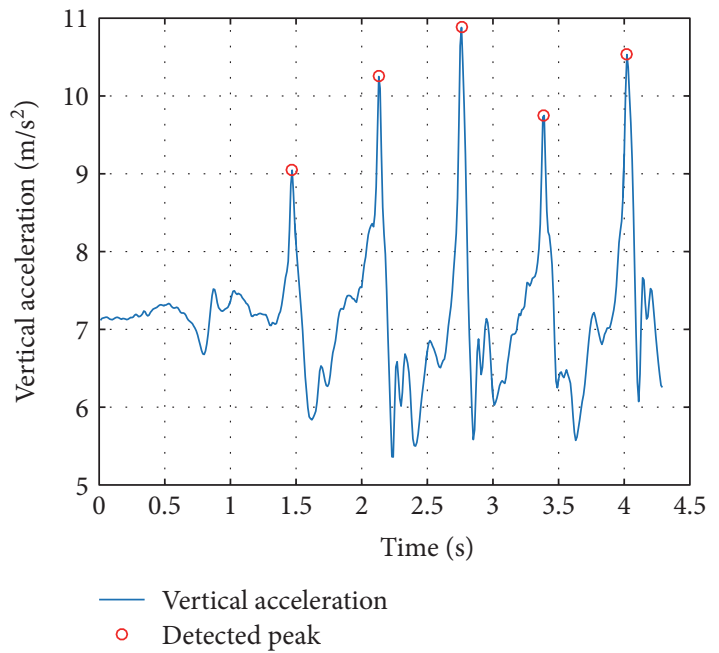

(b)

Figure 4: Peak detection of real walking data: (a) all peaks; (b) heel strike peaks.

data of sitting-standing up-standing-sitting down-sittingstanding up-standing-walking-running-walking-standingsitting down-sitting was recorded.

It is easy to classify the light activity among various activity and static cases. In this experiment, the parameters to detect static and vigor activity are 0.1 and 2, respectively. Other actions such as sitting down and standing up have the same standard deviation with walking but it can be eliminated since they happen in a short time while walking activity is periodic and occurs in a long time. Our next step is to detect the walking steps based on characteristics of walking activity (long and periodic occurrence).

There are many algorithms for step detection such as threshold level [19-21], spectral analysis [22], or peak detections. The threshold-based method in [19-21] detects a walking step when the acceleration value is larger than a threshold level. This method is simple but sensitive to noise since other acceleration could cause a fluctuation in the output of the sensor. Spectral analysis in [22] is robust to noise but has a large computational load.

To provide an online step detection that can run on low speed and limited memory processors, we propose a simple peak detection algorithm using five consecutive data points.

Let $y_{i} \in \mathbb{R}$ be a signal from the output of the sensor at the discrete time $i$. Since we are only interested in detecting the peak of the signal rather than its true values, $y_{i}$ is filtered by a simple three-point weighted moving average:

$$
\bar{y}_{i}=\alpha_{1} y_{i-1}+\left(1-2 \alpha_{1}\right) y_{i}+\alpha_{1} y_{i+1}
$$

where $\alpha_{1}$ is the weighting factor for the low-pass filter. The significant maximum peak at the discrete time $i$ will be detected if one of the following conditions is satisfied:

$$
\begin{gathered}
\bar{y}_{i-2}<\bar{y}_{i}, \\
\bar{y}_{i-1}<\bar{y}_{i}, \\
\bar{y}_{i}>\bar{y}_{i+1}, \\
\bar{y}_{i}>\bar{y}_{i+2}, \\
\bar{y}_{i}-\max \left(\bar{y}_{i-2}, \bar{y}_{i-1}, \bar{y}_{i+1}, \bar{y}_{i+2}\right)>\alpha_{2}, \\
\bar{y}_{i-3}<\bar{y}_{i-2}, \\
\bar{y}_{i-2}<\bar{y}_{i-1}, \\
\bar{y}_{i-1}<\bar{y}_{i}, \\
\bar{y}_{i}>\bar{y}_{i+1}, \\
\bar{y}_{i}-\max \left(\bar{y}_{i-3}, \bar{y}_{i-2}, \bar{y}_{i-1}, \bar{y}_{i+1}\right)>\alpha_{2}, \\
\bar{y}_{i-1}<\bar{y}_{i}, \\
\bar{y}_{i}>\bar{y}_{i+1}, \\
\bar{y}_{i+1}>\bar{y}_{i+2}, \\
\bar{y}_{i+2}>\bar{y}_{i+3}, \\
\bar{y}_{i}-\max \left(\bar{y}_{i-1}, \bar{y}_{i+1}, \bar{y}_{i+2}, \bar{y}_{i+3}\right)>\alpha_{2},
\end{gathered}
$$

where $\alpha_{2}$ is a threshold parameter. The sensitivity of the peak detection algorithm can be adjusted by changing the value of $\alpha_{2}$. If $\alpha_{2}$ is chosen as a large value, small peaks due to noise can be ignored. However, only conspicuous peaks can be recognized. This also means that some true peaks may not be detected.

Although all peaks are detected, there might be a chance that the peak is caused by other activities or noises rather than walking. For example, as can be seen from Figure 4(a) where an extraction of a fast walking data is from an accelerometer attached on a person's neck, if only the conditions in 


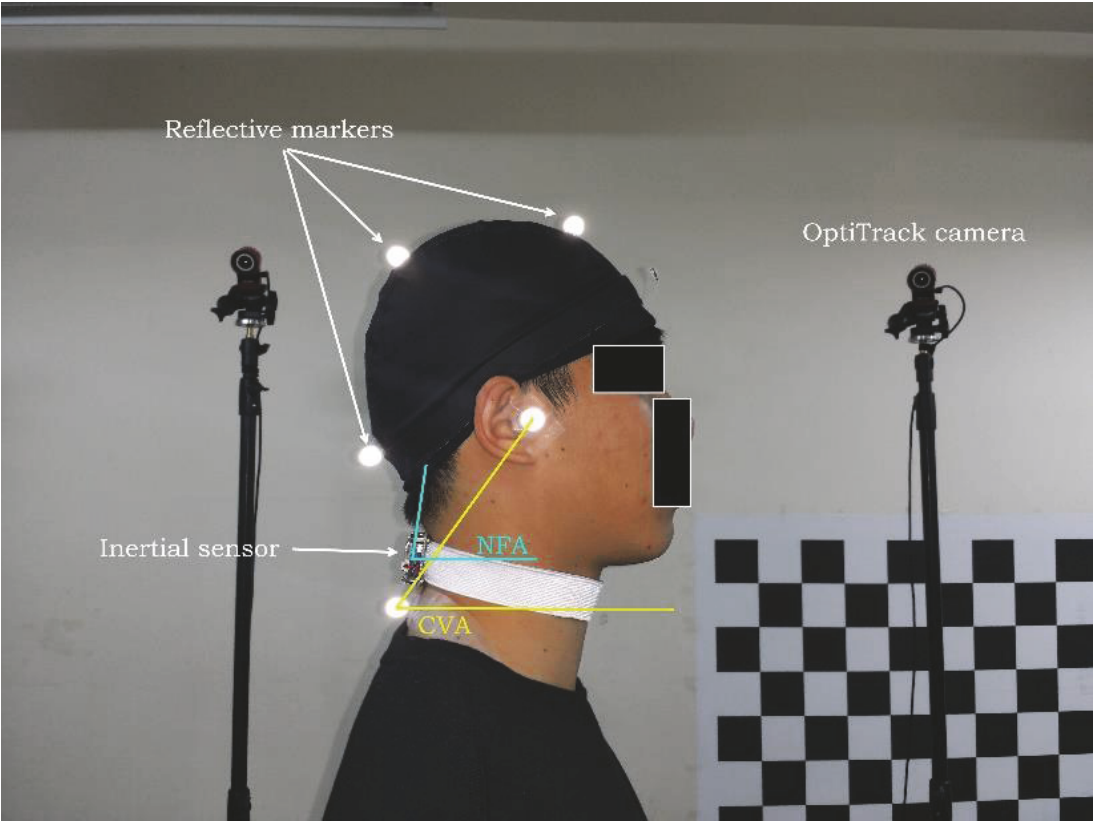

(a)

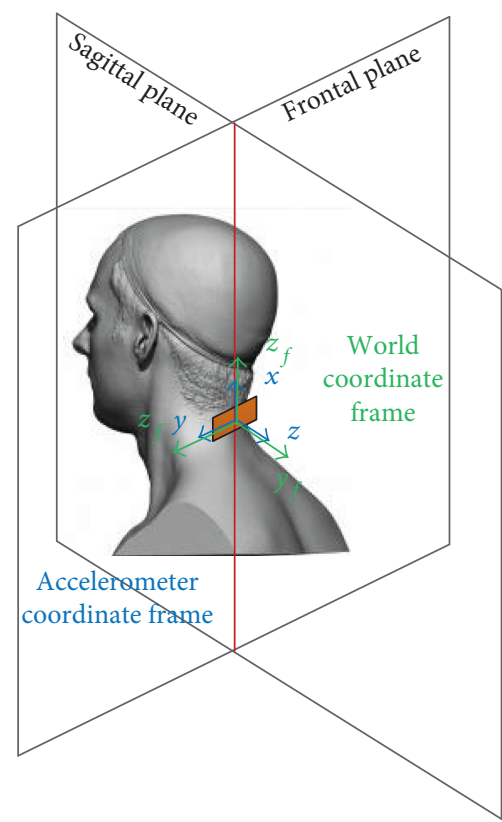

(b)

FIGURE 5: (a) Reflective markers and inertial sensor position while standing upright inside the working range of infrared camera system; (b) sensor and world coordinate frames used in this paper.

(3) are used, we obtain many peaks. Fortunately, peaks caused by the contacting shock at heel strike moments are much larger than other peaks and these peaks occur periodically. Based on this fact, we propose to detect the heel strike peeks by searching one large peak within a specific time $\alpha_{3}$. In detail, when a peak is detected at the time $i$ using conditions in (3), all surrounding peaks in the range of $\left[i-\alpha_{3}, i+\alpha_{3}\right]$ are compared together. The largest value will be the true peak generated by a heel strike. By using this method, periodic peaks will be selected.

The result of applying $\alpha_{3}$ condition is given in Figure 4(b) where all walking peaks are successfully detected. If $\alpha_{3}$ is too large, some steps will be missed. Conversely, if we set $\alpha_{3}$ as a small value, some peaks caused by noises will be wrongly detected as the walking peaks. However, the maximum value of $\alpha_{3}$ can be chosen from 0.4 to $0.6 \mathrm{sec}$ since the normal and fast walking speeds of a pedestrian is 100 and 150 steps per minute $[23,24]$, respectively. In this experiment, $\alpha_{3}$ is chosen to be $0.4 \mathrm{sec}$.

The walking activity is only defined when at least three consecutive walking peaks are detected. Let $T_{j}$ be the time interval between two consecutive peaks at $t_{i-1, j}$ and $t_{i, j}$ from $N$ peaks $(1 \leq j \leq N)$ :

$$
T_{j}=t_{i, j}-t_{i-1, j}
$$

Since walking activity is nearly periodic, the following condition will be satisfied:

$$
\left|T_{j}-\frac{1}{N} \sum_{k=1}^{N} T_{k}\right| \leq \alpha_{4} .
$$

If small $\alpha_{4}$ is chosen, we obtain very periodic walking steps but the walking activity may fail to be detected since naturally not all strike cycles are the same. In contrast, if a larger $\alpha_{4}$ is used, we have a more flexible condition in detecting walking activity. However, there could be a chance that some of the detected peaks are not the walking steps.

2.3. Neck Flexion Angle Estimation. The neck flexion angle (NFA), which is the angle of neck flexion relative to horizontal direction, is estimated by observing the angle between vertical direction and the projection of the gravitational force on the $z$-axis of the accelerometer. The accelerometer was attached on the body neck as shown in Figure 5(b) where its $x$-axis points upward and its $z$-axis is perpendicular to the neck when the neck is up straight. The world coordinate frame was chosen so that its $z$-axis is upward, while the $x$ - and $y$-axes can be chosen arbitrarily. When the neck is bended, the inclination angle of the neck can be calculated by using the following equation [25]:

$$
\beta=\tan ^{-1} \frac{a_{x}}{\sqrt{a_{y}^{2}+a_{z}^{2}}},
$$

where $a=\left[\begin{array}{lll}a_{x} & a_{y} & a_{z}\end{array}\right]^{T} \in \mathbb{R}^{3}$ is the output of the accelerometer.

Equation (6) is only applied for static cases where the external accelerations are absent. In walking scene, the external due to walking activity is considerable. As can be seen from Figure 1 and Figure 2(b), the projection of the gravitational force on the $x$ - and $z$-axes of the accelerometer are sinusoidal in each walking step. Since $x$-axis of the 
TABLE 1: CVA and NFA in static case (unit: degrees).

\begin{tabular}{|c|c|c|c|c|c|c|}
\hline Static case & $10 \mathrm{sec}$ no change & Level 1 & Level 2 & Level 3 & Level 4 & Level 5 \\
\hline Mean (NFA) & 83.6211 & 82.4773 & 76.2310 & 66.1847 & 58.4557 & 45.9955 \\
\hline Mean (CVA) & 49.3835 & 48.5494 & 40.7722 & 30.2919 & 22.7974 & 9.2151 \\
\hline Mean (NFA - CVA) & 34.2376 & 33.9280 & 35.4588 & 35.8928 & 35.6582 & 36.7804 \\
\hline Var (NFA - CVA) & 0.0632 & 0.0461 & 0.0518 & 0.0273 & 0.0340 & 0.0681 \\
\hline
\end{tabular}

accelerometer points upward while its $z$-axis is on walking direction, the inclination on the neck in each walking step can be estimated using the following equation:

$$
\beta=\tan ^{-1} \frac{\bar{a}_{x}}{\bar{a}_{z}}
$$

where $\bar{a}_{x}$ and $\bar{a}_{z}$ are the average value of accelerometer output in the $x$ and $z$ direction within each walking step, respectively.

\section{Experiments}

A total of 20 subjects, ranging from 24 to 32 years old with the mean age of 26.8 , were recruited for the study. All participants had no difficulties in using smartphones while standing or walking and had at least 15 minutes walking to be familiar with the treadmill (running machine).

We conducted all trials with participants in two separated experiments.

During the whole process, subjects were asked to wear a swimming cap having three reflective markers attached in the sagittal plane parallel to the direction of view. Two more markers were placed on the skin at the position of C7 bone and tragus.

An Xsens MTil sensor with the customized board having Bluetooth communication was put on subjects' neck using an elastic band. Both two experiments were conducted inside the working range of 6 cameras in the OptiTrack infrared camera system (Figure 5(a)). The system was configured to extract 3D position data of the markers, 3-axis acceleration, and angular velocity of the inertial sensor at the sampling rate of $100 \mathrm{~Hz}$.

At the beginning of the first experiment, each subject's data was recorded while they were standing up straight (upright position) as they usually did for 10 seconds. Then each participant intentionally performed "text-neck" posture [26] changes with 5 levels from upright position and the lowest position within about 10 seconds each. These levels were chosen by each subject, whichever make themselves comfortable.

Before the second experiment started, participants were asked to get acquainted with different speed levels of the treadmill. Each subject then sequentially performed walking tasks with regular constant walking speed of 3, 4, and $5 \mathrm{~km} / \mathrm{h}$. During each task, the subject also did the 5 levels of "text-neck" changes, each level within 30 seconds. At least 30 seconds of rest break was given after finishing each task to minimize the risk of neck pain.
Position data of markers and output of inertial sensor were synchronized before using for analysis. The craniovertebral angle (CVA) and the neck flexion angle (NFA or the neck angle) can be derived using the following procedure:

(a) The CVA, which is the angle between a line connecting $\mathrm{C} 7$ to tragus and the horizontal line through the spinous process of $\mathrm{C} 7$ [27] can be calculated by projection of both $\mathrm{C} 7$ and tragus markers into the sagittal plane.

(b) The neck angle NFA in the static pose can be calculated by observing the angle between the vertical direction and the projection of the gravitational force at each sample of time on the $z$-axis of the accelerometer using (6), which is called "direct computation method." However, in the walking case, the center of mass has sinusoidal movements in lateral and vertical direction [16], the external acceleration in that two directions are close to sinusoidal forms. The forward acceleration is approximately zero (linear forward walking), then we can eliminate the external accelerations by summing the accelerometer output in each axis within each walking step, and the inclination angle with respect to the ground can be calculated using (7).

To evaluate the relationship between the CVA and the NFA, we inspected the mean and variation of the difference between that two angles. Our estimation algorithm performance is also examined by comparing with the "direct computation method" and "fixed interval average method," where the acceleration data in each axis is summed over a fixed period. We chose that fixed interval to be $0.5,0.75$, and 1 second due to the common walking step time.

\section{Results and Discussions}

The main contributions of this study include the relationship between CVA and NFA in both static and walking cases. The experimental results of a subject showed in Tables 1 and 2 imply that the estimated neck angles (NFA) have a linear relationship with the CVA, where there is a "nonchanging" offset value between the two angles in both static and walking cases. Also in Figure 6, we can clearly see the similar trends and amount of changes between different levels among these angles. It means the NFA, which can be derived by our simple, flexible, and cost-effective method, can be used instead of the CVA in applications, and from the estimated angle and a calibration step, the true neck angle can be 
TABLE 2: CVA and NFA in walking case (unit: degrees).

\begin{tabular}{|c|c|c|c|c|c|c|}
\hline & Walking case & Level 1 & Level 2 & Level 3 & Level 4 & Level 5 \\
\hline \multirow{4}{*}{$3 \mathrm{~km} / \mathrm{h}$} & Mean (NFA) & 81.4722 & 74.4427 & 71.6546 & 65.7486 & 58.8117 \\
\hline & Mean (CVA) & 45.3579 & 37.3134 & 34.1081 & 28.1364 & 21.5184 \\
\hline & Mean (NFA - CVA) & 36.1144 & 37.1292 & 37.5465 & 37.6123 & 37.2933 \\
\hline & Var (NFA - CVA) & 0.3312 & 0.1863 & 0.1491 & 0.3100 & 0.5335 \\
\hline \multirow{4}{*}{$4 \mathrm{~km} / \mathrm{h}$} & Mean (NFA) & 82.7458 & 75.8507 & 69.3977 & 65.0855 & 60.2854 \\
\hline & Mean (CVA) & 46.0182 & 38.7697 & 31.8672 & 27.5250 & 22.9371 \\
\hline & Mean (NFA - CVA) & 36.7277 & 37.0810 & 37.5305 & 37.5605 & 37.3483 \\
\hline & Var (NFA - CVA) & 0.9034 & 0.4096 & 0.7186 & 0.8024 & 0.5123 \\
\hline \multirow{4}{*}{$5 \mathrm{~km} / \mathrm{h}$} & Mean (NFA) & 82.0928 & 74.7623 & 69.1052 & 64.1363 & 58.7212 \\
\hline & Mean (CVA) & 45.9104 & 37.4357 & 31.0791 & 26.0616 & 21.2336 \\
\hline & Mean (NFA - CVA) & 36.1824 & 37.3266 & 38.0261 & 38.0747 & 37.4876 \\
\hline & Var (NFA - CVA) & 0.2328 & 0.3207 & 0.1990 & 0.4291 & 0.6641 \\
\hline
\end{tabular}

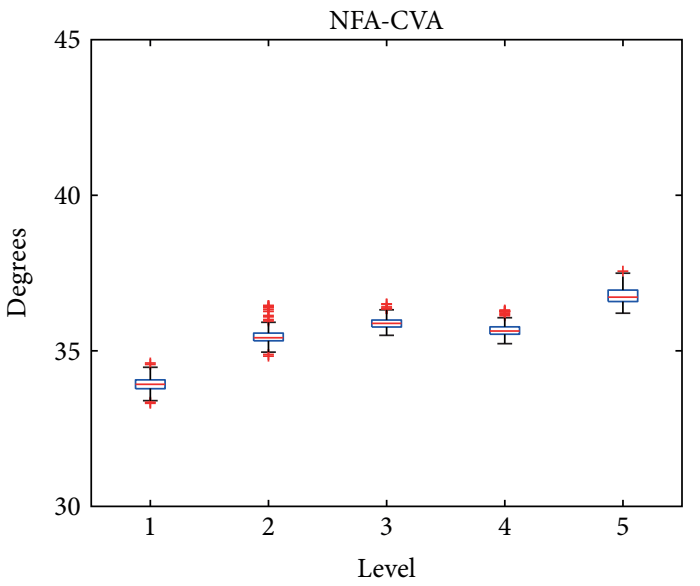

(a)

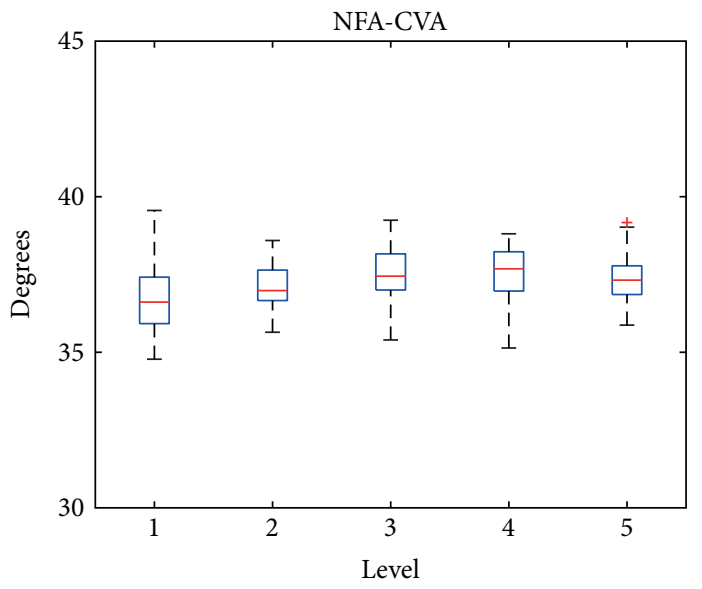

(c)

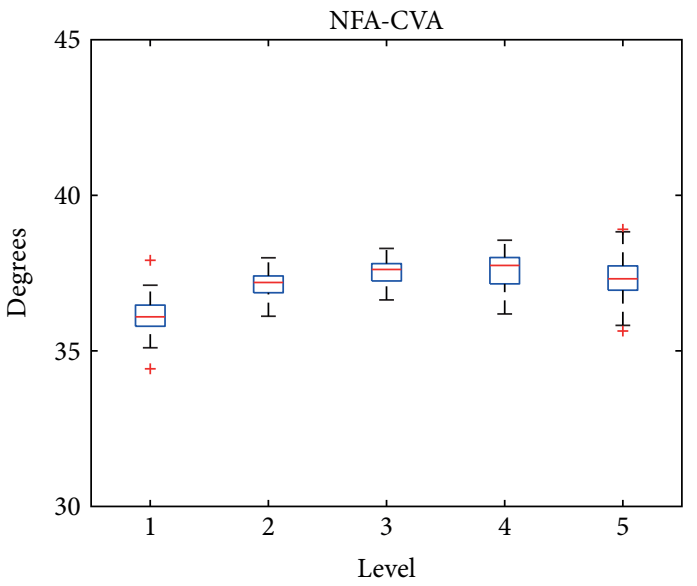

(b)

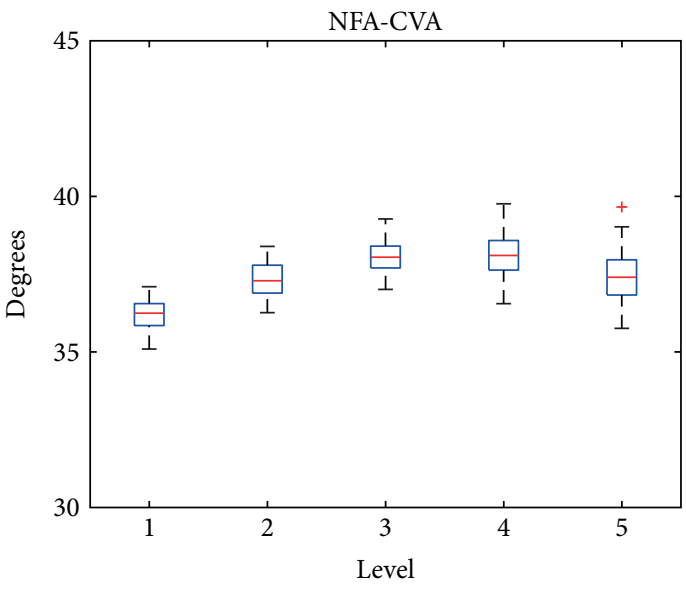

(d)

Figure 6: Relationship between CVA and NFA in (a) static case; (b) $3 \mathrm{~km} / \mathrm{h}$ walking; (c) $4 \mathrm{~km} / \mathrm{h}$ walking; (d) $5 \mathrm{~km} / \mathrm{h}$ walking.

derived. The calibration step can be done using the following simple process:

(a) The calibrated angle is obtained when the candidate sits upright. (b) This upright angle is assumed to be 90 degrees for the estimated neck angle at that moment.

(c) The difference between the real estimated and upright angle is added into each estimated neck angle value. 
TABLE 3: Mean of absolute error of the estimated neck angle after calibration steps (unit: degrees).

\begin{tabular}{|c|c|c|c|c|c|c|}
\hline \multicolumn{2}{|c|}{ Walking case } & \multirow{2}{*}{$\frac{\text { Direct computation method }}{1.8637}$} & \multirow{2}{*}{$\begin{array}{c}\text { Fixed interval } \\
T=0.5 \mathrm{sec} \\
0.5419\end{array}$} & \multirow{2}{*}{$\begin{array}{c}\text { Fixed interval } \\
T=0.75 \mathrm{sec}\end{array}$} & \multirow{2}{*}{$\begin{array}{c}\text { Fixed interval } \\
T=1 \mathrm{sec} \\
0.4982\end{array}$} & \multirow{2}{*}{$\begin{array}{c}\text { Proposed flexible interval } \\
0.4836\end{array}$} \\
\hline \multirow{6}{*}{$3 \mathrm{~km} / \mathrm{h}$} & Level 1 & & & & & \\
\hline & Level 2 & 1.8715 & 0.5579 & 0.5356 & 0.4999 & 0.4826 \\
\hline & Level 3 & 1.7772 & 0.5449 & 0.4932 & 0.4780 & 0.4545 \\
\hline & Level 4 & 1.8539 & 0.5632 & 0.5650 & 0.4928 & 0.4793 \\
\hline & Level 5 & 2.0296 & 0.6462 & 0.6297 & 0.5337 & 0.5074 \\
\hline & Mean & 1.8792 & 0.5708 & 0.5528 & 0.5005 & 0.4815 \\
\hline \multirow{6}{*}{$4 \mathrm{~km} / \mathrm{h}$} & Level 1 & 2.4324 & 0.7696 & 0.7460 & 0.8390 & 0.6590 \\
\hline & Level 2 & 2.2248 & 0.6280 & 0.6395 & 0.5494 & 0.5222 \\
\hline & Level 3 & 2.2666 & 0.7383 & 0.6646 & 0.6376 & 0.5636 \\
\hline & Level 4 & 2.2077 & 0.8018 & 0.5846 & 0.6797 & 0.5552 \\
\hline & Level 5 & 2.2607 & 0.6116 & 0.6482 & 0.6276 & 0.5534 \\
\hline & Mean & 2.2784 & 0.7099 & 0.6566 & 0.6667 & 0.5707 \\
\hline \multirow{6}{*}{$5 \mathrm{~km} / \mathrm{h}$} & Level 1 & 2.6015 & 0.5970 & 0.6359 & 0.5893 & 0.5434 \\
\hline & Level 2 & 2.5488 & 0.6479 & 0.6007 & 0.5484 & 0.5537 \\
\hline & Level 3 & 2.3671 & 0.5444 & 0.5038 & 0.5273 & 0.4856 \\
\hline & Level 4 & 2.3915 & 0.5932 & 0.6272 & 0.6478 & 0.5585 \\
\hline & Level 5 & 2.5111 & 0.6832 & 0.6684 & 0.7290 & 0.5866 \\
\hline & Mean & 2.4840 & 0.6131 & 0.6072 & 0.6084 & 0.5456 \\
\hline
\end{tabular}

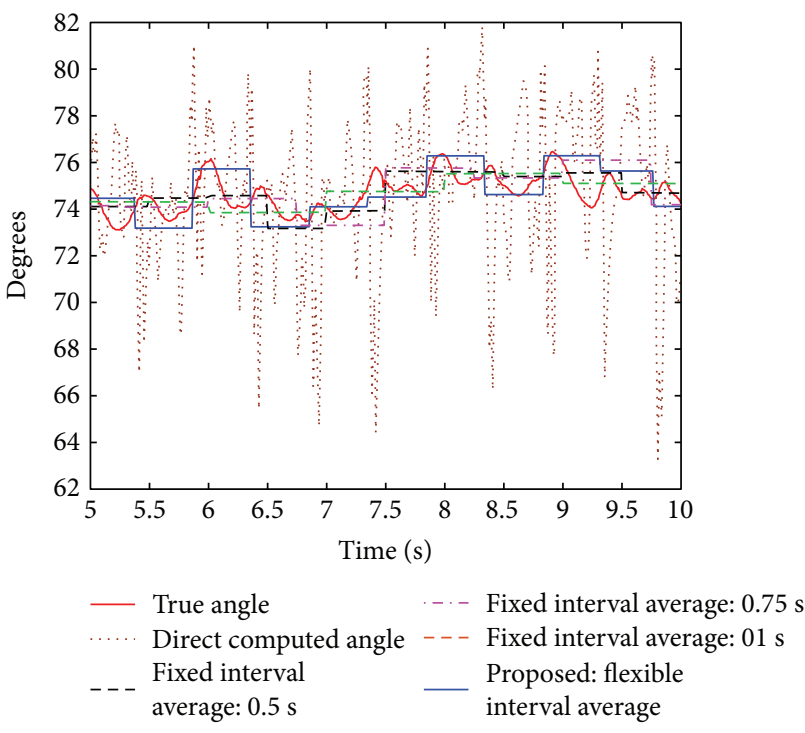

Figure 7: Estimated neck angle of different methods at $3 \mathrm{~km} / \mathrm{h}$.

Experiment data from all candidates were also included to examine the performance of our proposed neck angle estimation method. Table 3 shows the mean of absolute errors of the estimated neck angle which belong to the "direct computation method" (calculate angles at each sample of data), our proposed walking step time-based "flexible interval average method" (calculate angles based on average of acceleration between consecutive walking steps), and the "fixed interval average method" (calculate angles based on average of acceleration during a fixed time interval of 0.5 ,
0.75 , and 1 second). The true neck flexion angle (NFA) was obtained through a calibration step from the craniovertebral angle (CVA) measured by OptiTrack camera system. An example of visual comparison between methods above is presented in Figure 7.

The result from Table 3 shows that our proposed method provides better result in all contexts in comparison with others. More specifically, the "direct computation method" at each sample of data gives an acceptable result with mean of absolute error of estimated angle varying from 1.88 to 2.49 degrees. Since the external accelerations in forward and vertical directions can be canceled out by taking average in each walking step, our proposed method presents better result in comparison to other methods with mean of absolute error from 0.48 to 0.57 degrees. Moreover, the walking step length and the human gait while walking at each speed are personal dependent parameters, and the proposed method shows its flexibility that we can use for personal independent parameters.

\section{Conflicts of Interest}

The authors declare no conflict of interest.

\section{Authors' Contributions}

Duc Cong Dang, Quoc Khanh Dang, Young Joon Chee, and Young Soo Suh conceived and designed this study. Duc Cong Dang and Quoc Khanh Dang performed the experiments. Duc Cong Dang wrote the paper. Young Soo Suh reviewed and edited the manuscript. 


\section{Acknowledgments}

This work was supported by National Research Foundation of Korea grant funded by the Korean Government (no. 2016R1D1A1A09918657).

\section{References}

[1] S. P. Cohen, "Epidemiology, diagnosis, and treatment of neck pain," Mayo Clinic Proceedings, vol. 90, no. 2, pp. 284-299, 2015.

[2] K. K. Hansraj, "Assessment of stresses in the cervical spine caused by posture and position of the head," Surgical Technology International, vol. 25, pp. 277-279, 2014.

[3] G. P. Szeto, L. Straker, and S. Raine, "A field comparison of neck and shoulder postures in symptomatic and asymptomatic office workers," Applied Ergonomics, vol. 33, no. 1, pp. 75-84, 2002.

[4] L. Korpinen, R. Paakkonen, and F. Gobba, "Self-reported neck symptoms and use of personal computers, laptops and cell phones among Finns aged 18-65," Ergonomics, vol. 56, no. 7, pp. 1134-1146, 2013.

[5] J. E. Gold, J. B. Driban, N. Thomas, T. Chakravarty, V. Channell, and E. Komaroff, "Postures, typing strategies, and gender differences in mobile device usage: an observational study," Applied Ergonomics, vol. 43, no. 2, pp. 408412, 2012.

[6] X. Guan, G. Fan, Z. Chen et al., "Gender difference in mobile phone use and the impact of digital device exposure on neck posture," Ergonomics, vol. 59, no. 11, pp. 1453-1461, 2016.

[7] S. Lee, H. Kang, and G. Shin, "Head flexion angle while using a smartphone," Ergonomics, vol. 58, no. 2, pp. 220-226, 2015.

[8] C. H. Basch, D. Ethan, P. Zybert, and C. E. Basch, "Pedestrian behavior at five dangerous and busy Manhattan intersections," Journal of Community Health, vol. 40, no. 4, pp. 789-792, 2015.

[9] S. Haga, A. Sano, Y. Sekine, H. Sato, S. Yamaguchi, and K. Masuda, "Effects of using a smart phone on pedestrians' attention and walking," Procedia Manufacturing, vol. 3, pp. 2574-2580, 2015.

[10] C. Demmans, S. Subramanian, and J. Titus, "Posture monitoring and improvement for laptop use," in In CHI '07 Extended Abstracts on Human Factors in Computing Systems, pp. 2357-2362, ACM, San Jose, CA, USA, 2007.

[11] L. M. Straker, J. Coleman, R. Skoss, B. A. Maslen, R. BurgessLimerick, and C. M. Pollock, "A comparison of posture and muscle activity during tablet computer, desktop computer and paper use by young children," Ergonomics, vol. 51, no. 4, pp. 540-555, 2008.

[12] J. G. Young, M. Trudeau, D. Odell, K. Marinelli, and J. T. Dennerlein, "Touch-screen tablet user configurations and case-supported tilt affect head and neck flexion angles," Work, vol. 41, no. 1, pp. 81-91, 2012.

[13] S. Jeong, T. Song, H. Kim, M. Kang, K. Kwon, and J. W. Jeon, "Human neck's posture measurement using a 3-axis accelerometer sensor," in Computational Science and its Applications - ICCSA 2011: International Conference, pp. 96-109, Santander, Spain, June 2011, Proceedings, part V.

[14] P. P. Breen, A. Nisar, and G. Olaighin, "Evaluation of a single accelerometer based biofeedback system for real-time correction of neck posture in computer users," 2009 Annual
International Conference of the IEEE Engineering in Medicine and Biology Society, 2009, pp. 7269-7272, Minneapolis, MN, USA, 2009.

[15] D. C. Dang, Q. K. Dang, Y. J. Chee, and Y. S. Suh, "Motion sensor based neck posture estimation during walking," in Proceedings of 22nd International Symposium on Artificial Life and Robotics (AROB), pp. 904-908, Beppu, Japan, January 2017.

[16] M. S. Orendurff, A. D. Segal, G. K. Klute, J. S. Berge, E. S. Rohr, and N. J. Kadel, "The effect of walking speed on center of mass displacement," Journal of Rehabilitation Research \& Development, vol. 41, no. 6, pp. 829-834, 2004.

[17] Carnegie Mellon University, "Cmu graphics lab motion capture database," NSF Grant \#0196217, May 2017, http:// mocap.cs.cmu.edu.

[18] H. D. Black, "A passive system for determining the attitude of a satellite,” AIAA Journal, vol. 2, no. 7, pp. 1350-1351, 1964.

[19] Y. W. Bai, C. H. Yu, and S. C. Wu, "Using a three-axis accelerometer and gps module in a smart phone to measure walking steps and distance," in Electrical and Computer Engineering (CCECE), 2014 IEEE 27th Canadian Conference, pp. 1-6, Toronto, ON, Canada, May 2014.

[20] T. Kinh, L. Tu, and D. Tien, "A high-accuracy step counting algorithm for iphones using accelerometer," in 2012 IEEE International Symposium on Signal Processing and Information Technology (ISSPIT), pp. 213-217, Ho Chi Minh City, Vietnam, 2012.

[21] M. Yamamoto and E. Kamioka, "Location Estimation System using Acceleration Sensor," The Institute of Electronics, Information, and Communication Engineers Technical Report, Mobile Network and Applications, vol. 110, no. 40, pp. 139144, 2010.

[22] H. Ookura, H. Yamamoto, and K. Yamazaki, "Development and evaluation of walking path estimation system using sensors of android device and vector map matching," in The International Conference on Information Network, pp. 25-29, Bali, Indonesia, February 2012.

[23] M. Stanten, "Stepping up fitness: walking 5 miles a day is easier than you think!," November 2011, https://www.prevention .com/fitness/fitness-tips/stepping-fitness.

[24] S. J. Marshall, S. S. Levy, C. E. Tudor-Locke et al., "Translating physical activity recommendations into a pedometer-based step goal: 3000 steps in 30 minutes," American Journal of Preventive Medicine, vol. 36, no. 5, pp. 410-415, 2009.

[25] K. Tuck, Tilt Sensing Using Linear Accelerometers, Freescale Semiconductor: Application Note AN3461, Tempe, AZ, 2007.

[26] S. Shoshany, "A Modern Spine Ailment: Text Neck," November 2015, http://www.spine-health.com/blog/modern-spineailment-text-neck.

[27] P. Nejati, S. Lotfian, A. Moezy, A. Moezy, and M. Nejati, "The relationship of forward head posture and rounded shoulders with neck pain in Iranian office workers," Medical Journal of the Islamic Republic of Iran, vol. 28, p. 26, 2014. 


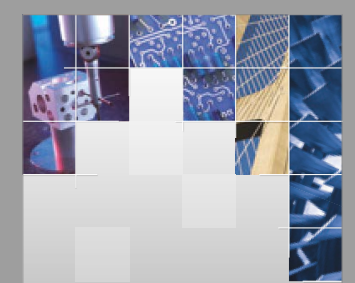

\section{Enfincering}
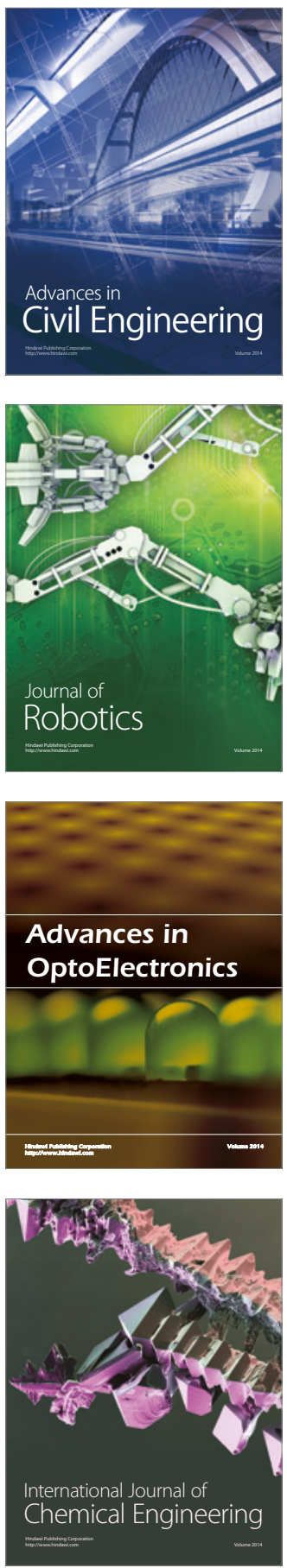

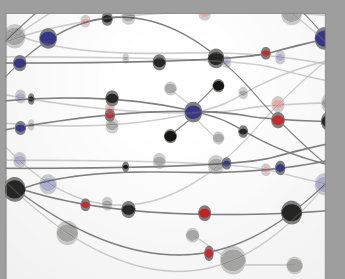

The Scientific World Journal

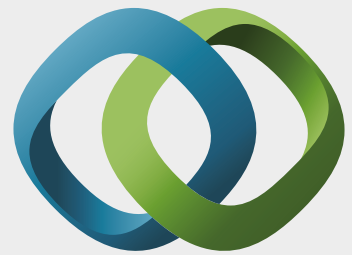

\section{Hindawi}

Submit your manuscripts at

https://www.hindawi.com
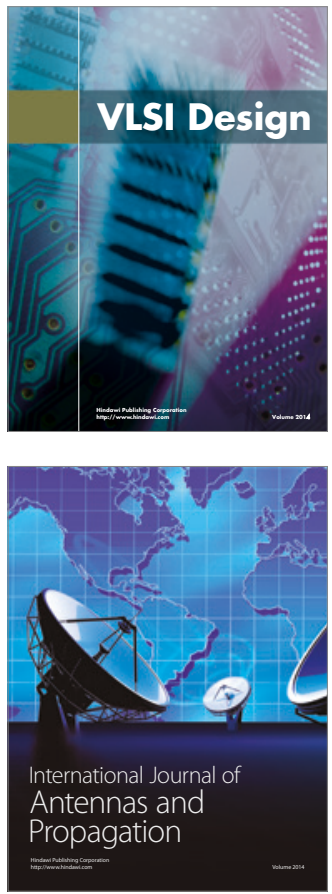

\section{Rotating}

Machinery
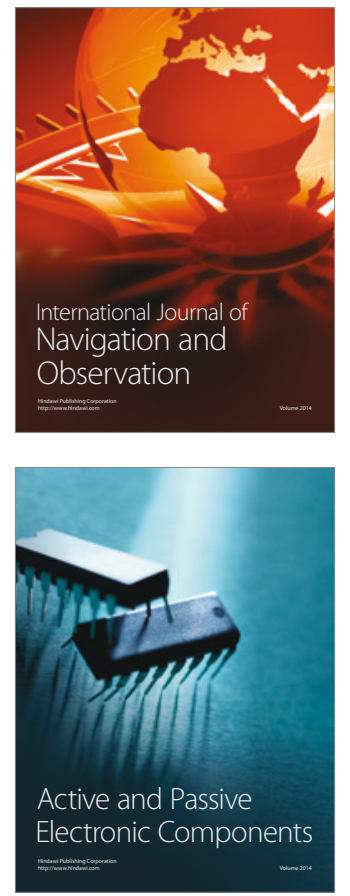
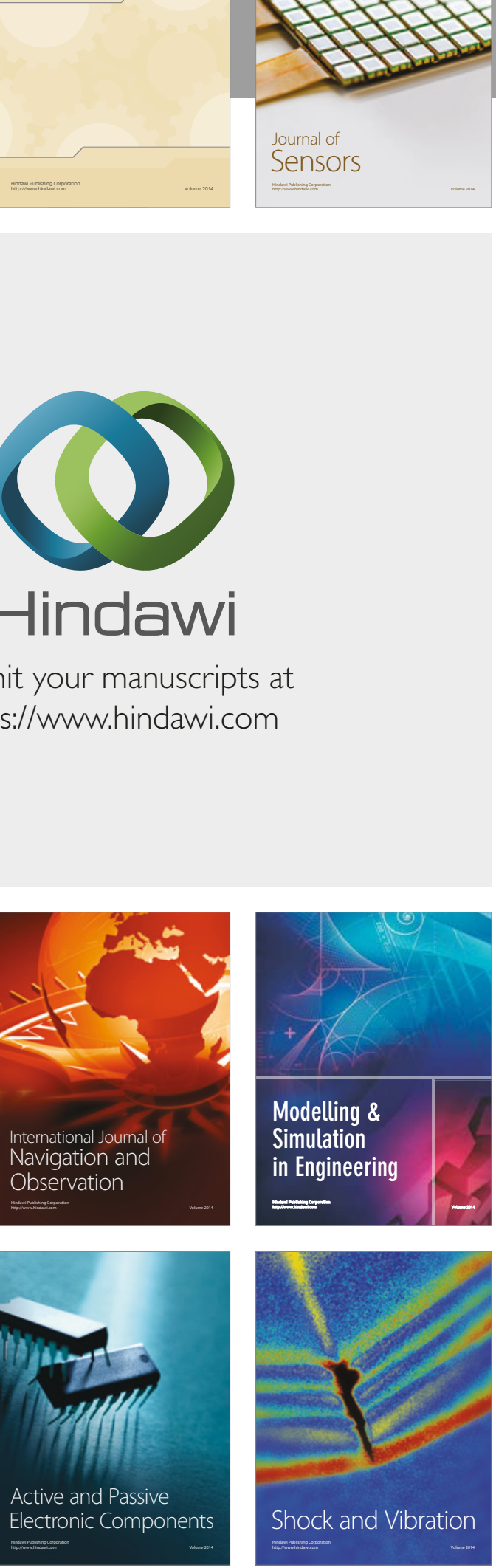
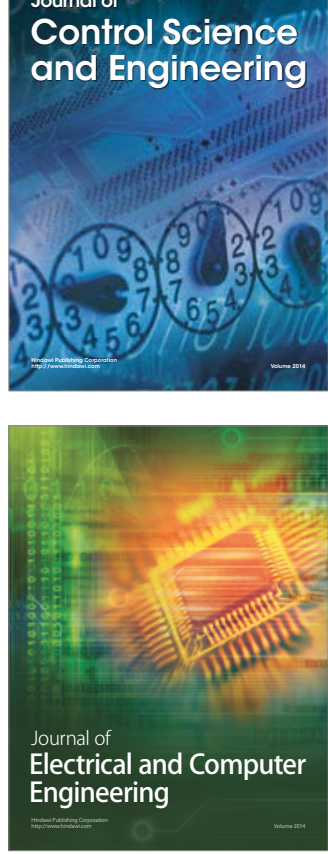

Distributed

Journal of

Control Science

and Engineering
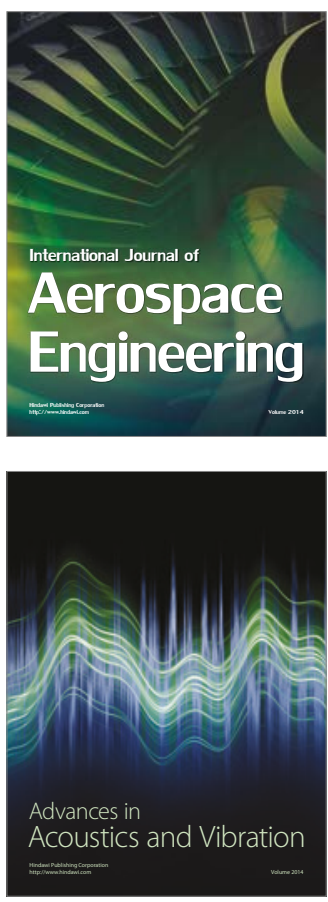

Sensor Networks 